\title{
Prevalence and Factors Associated with Major Depression Among Female Sex Workers in Post- Conflict Gulu District: A Cross-Sectional Study
}

Ouma Simple ( $\square$ oumasimple@gmail.com )

The AIDS Support Organisation (TASO) https://orcid.org/0000-0002-4510-6736

Nazarius Mbona Tumwesigye

Makerere University College of Health Sciences

Rawlance Ndejjo

Makerere University College of Health Sciences

Catherine Abbo

Makerere University School of Medicine

Research article

Keywords: depression, female sex workers, Gulu district, post-conflict setting

Posted Date: August 3rd, 2020

DOI: https://doi.org/10.21203/rs.3.rs-50097/v1

License: (a) (i) This work is licensed under a Creative Commons Attribution 4.0 International License.

Read Full License 


\section{Prevalence and factors associated with major depression among female sex workers in post-conflict Gulu district: a Cross-Sectional Study}

Authors: Ouma Simple ${ }^{1,2^{*}}$, Nazarius Mbona Tumwesigye ${ }^{2}$, Rawlance Ndejjo ${ }^{3}$, Catherine Abbo $^{4}$

\section{Authors details and affiliations}

${ }^{1}$ Department of Epidemiology and Biostatistics, School of Public Health, College of Health Sciences, Makerere University, Kampala, Uganda

${ }^{2}$ Department of Research, The AIDS Support Organization (TASO), TASO Headquarters, Kampala, Uganda.

${ }^{3}$ Department of Disease Control and Environmental Health School of Public Health, College of Health Sciences, Makerere University, Kampala, Uganda

${ }^{4}$ Department of Psychiatry, School of Medicine, College of Health Sciences, Makerere University, Kampala, Uganda

*Correspondence: oumasimple@gmail.com or oumas@ tasouganda.org 


\begin{abstract}
Background: Depression is the world's leading cause of disability and a major contributor to the overall global burden of disease. Female sex workers operating in conflict-affected settings could be more prone to depression, yet the prevalence and factors associated with depression among this special population remain inadequately addressed. This study thus aimed to determine the prevalence and factors associated with major depression among female sex workers in postconflict Gulu district.
\end{abstract}

Methods: This cross-sectional study was conducted among 300 randomly selected female sex workers in Gulu district between March and June 2020. Possible factors associated with depression were assessed using pre-tested semi-structured questionnaire and participants assessed for major depression using Mini-International Neuropsychiatric Interview (MINI) version 7.0.0. We utilized STATA 14.0 for univariate, bivariate and multivariate data analyses.

Results: The mean age of study participants was 26.4 years (SD \pm 6 ), 57.7\% attained primary education, $51.7 \%$ never married and $42.1 \%$ were living with HIV. Almost half $(47.7 \%)$ of the participants had major depression, of whom half $(50.4 \%)$ had severe depression and one-third (34.3\%) had suicidal ideation. At multivariate level, factors that showed positive association with major depression were: psychological stressor $(\mathrm{aOR}=11.0,95 \% \mathrm{CI}$ : 5.77-20.86), living with HIV $(\mathrm{aOR}=2.32,95 \% \mathrm{CI}: 1.29-4.15)$, being verbally abused $(\mathrm{aOR}=2.27,95 \% \mathrm{CI}: 1.26-4.07)$, and being $30+$ years of age $(\mathrm{aOR}=1.95,95 \% \mathrm{CI}: 1.05-3.61)$. Meanwhile, factors that showed negative association with major depression were: providing sexual services at client's place $(\mathrm{aOR}=0.46$, 95\%CI: 0.24-0.91), using modern family planning method (aOR=0.47, 95\%CI: 0.25-0.87), and daily intake of alcohol (aOR=0.50, 95\%CI: 0.28-0.88).

Conclusion: The findings underscore the high prevalence of major depression among female sex workers driven by psychological stress, living with HIV/AIDs, being verbally abused by clients and being 30+years of age. The high prevalence of major depression has important public health ramifications at individual and societal levels and requires urgent attention of all stakeholders.

Key words: depression, female sex workers, Gulu district, post-conflict setting 


\section{Background}

Depression is the world's leading cause of disability and a major contributor to the overall global burden of disease affecting more than 300 million people (1). Major depression (MD) can cause profound disability and death through suicide as well as through causing or worsening pre-existing physical illnesses (2). Globally, the disease burden from depression accounts for $32.4 \%$ of years lived with disability and $13.0 \%$ of disability-adjusted life-years (3). Annually, the costs per case of depression (in terms of treatment, morbidity and mortality) is in the range of $£ 3500-£ 6600$. The biggest portion of the costs is due to loss of productivity (4). Depression shows a bi-directional relationship with physical illnesses. Indeed, depression can increase one's risk for acquiring physical illnesses like HIV infections and contribute to physical injury like self-harms and motor accidents. Conversely, suffering from physical illnesses, especially the chronic diseases such as diabetes mellitus and HIV can increase one's risk of acquiring depressive disorders (5).

There are several known risk factors for major depression (MD) in the general population including biological factors such as chronic physical illness, experience of traumatic life events like rape, loss of loved ones, social adversity like extreme poverty and demographic factor for example being female (6)(7). Among FSWs, risk factors for depression include: experience of sexual, physical or psychological violence and the psychological and physical burden of sex work itself (8). In addition, many FSWs are exposed to alcohol and illicit drug use (9) which may cause physical illnesses and/or problem that eventually lead to development of MD (10).

Suffering from MD can lead to serious negative health and socioeconomic impacts on the affected individuals, families and society. Among sex workers, depressive symptoms can reduce one's level of sexual satisfaction (11). Yet the use of anti-depressants can further lead to development or worsening of sexual dysfunction (12). Additionally, untreated MD in FSWs can impede the progress made towards prevention of HIV, other sexually transmitted infections (STIs), and unwanted pregnancies since FSWs with MD are less likely to use condoms during sexual intercourse (13). Moreover, among the HIV positive FSWs, MD can lead to poor adherence to antiretroviral therapy (ART), unsuppressed viral load and subsequent transmission of HIV infection to their clients and children (14). Moreover, HIV-negative FSWs with MD have diminished ability to negotiate for safer sex with their partners and can easily become victim of sexual violence like rape and other risky sexual behaviours (15).

People living in conflict-affected settings are more prone to MD resulting from their experience of traumatic life events and the negative socioeconomic circumstances due to conflict (16)(17). The risk of MD could be worst for FSWs in Uganda because in the country, sex work is an illegal and prosecutable crime. Additionally, sex workers are not legally protected like people in recognized employments (18). The illegal nature of sex work along the intolerable culture and religious beliefs in the country could easily lead to high level of stigma, GBV and depression among FSWs. Primary prevention of depression is not feasible but secondary prevention through early diagnosis and management can be cost-saving. Secondary prevention of depression can only be possible if we can reduce cases of undiagnosed and under treated MD (19). Moreover, when treated promptly and effectively, many of the negative effects of depression on the lives and work of FSWs can be averted. Therefore, our study was aimed at determining the prevalence and factors associated with MD among FSWs in post-conflict Gulu district so as to generate information necessary to inform appropriate interventions to reduce the burden of MD among FSWs. 


\section{Methods}

\section{Study setting}

The study was conducted in Gulu, a district in Northern Uganda, an area that severely suffered from more than 20 years of the Lord's Resistant Army (LRA) rebellion between 1986 and 2007. Since the end of the war, the district has been under peace recovery program that has been rather slow and without meaningful economic resettlement package to the individuals and families affected by the conflict leaving many individuals including the FSWs struggling for survival. More than $80 \%$ of the inhabitants of the district practice subsistence farming (20). Gulu district has an estimated 1425 FSWs (21), majority of whom work and live within Gulu municipality. Unpublished program data show that more than 1300 fully mapped-out FSWs receive either HIV treatment or HIV preventive services from TASO Gulu.

\section{Study design and population}

We conducted a cross-sectional study among 300 active adult FSWs aged 18+ years and operating within Gulu district.

\section{Sample size and sampling}

Data were collected among 300 FSWs between March and June 2020. The sample size was determined using the Cochran [1963,1975] (22) formula: $\mathrm{n}_{0}=\mathrm{Z}^{2} \mathrm{pq} / \mathrm{e}^{2}$. At 95\% confidence level, $5 \%$ precision level, and when the proportion of FSWs in Uganda with depressive symptoms is $27 \%$ (23), the calculated sample size was 303 . However, we adjusted the sample size by $20 \%$ to cater for mobility $(10 \%)$ and non-response $(10 \%)$, the adjusted sample size became 380. The participants were selected using simple random sampling since their up to date details (database) were available from the key populated program data at TASO Gulu. However, the database was rapidly updated with the help of peers of FSWs before sampling so that new arrivals were included.

\section{Data collection}

Data on the independent variables like socio-demographic characteristics, socio-economic characteristics, sexual and reproductive health characteristics, condom use, alcohol and other illicit drug use were collected through face-to-face interviews using the pre-tested semi-structured questionnaire with imbedded MINI 7.00 developed in English language and translated into Acholi language (Luo) which is the predominantly spoken language in Gulu district. Data were collected by the first author and a trained female research assistant in either Acholi or English language as guided by participant's literacy level and preference.

\section{Data management and statistical analysis}

Data collected were entered and cleaned in EPI INFO 7 and then exported to STATA 14.0 for analysis. The diagnosis of MD, the dependent variable, was made using MINI 7.0.0, a tool based on DSM-5 diagnostic criteria (24). Diagnosis of MD was made when a participant had at least 2 weeks of persistent depressed mood and/or hopelessness, plus additional symptoms from MD diagnostic criterion A, for a total of 5 of the 9 DSM-5 criteria for MD (25). In addition, the symptoms of MD must have caused significant distress or problem and significantly altered ones behaviour and/or functionality. As guided by previous study, MD was classified based on severity 
as; mild if there were only 5 symptoms (the minimum needed for diagnosis of MD), moderate if there were 6-7 symptoms, and severe if there were 8-9 symptoms (26). Univariate analyses were described using either frequencies with their corresponding proportions for categorical variables or means with corresponding standard deviations for continuous variables. Bivariate analysis was conducted using simple logistic regression. Results of bivariate analyses were described using unadjusted odd ratios (uOR) with corresponding confidence intervals and p-values. Independent variables with a $\mathrm{p}<0.20$ were included in the multivariable analysis which was conducted using multivariate logistic regression with backward elimination until a final "best fit" model was derived. Prior to fitting the multivariable logistic regression model, eligible variables were checked for multicollinearity. When two variables showed multicollinearity $(r \geq 0.4)$, only one of them was included in the model. All eligible independent variables were entered at the beginning step of model building. Then, to avoid the model being over fitted, all non-significant variables were removed in a stepwise manner until only significant variables were left. The key outputs from multivariable analyses were adjusted odd ratio (aOR) with their corresponding $95 \%$ confidence intervals (CI) and p-values. Predictors from multivariate analyses whose CI did not include the null value (1.0) were considered statistically significant.

Regression diagnostics tests were conducted after fitting the multivariate logistic regression model. The model was checked for outliers and influential points using the predicted residuals. The predictive power of the model was also investigated using the sensitivity and specificity analyses. The Hosmer-Lemeshow's goodness-of-fit test was also performed to ascertain how the model was fitting the data without omitting important variables that could cause bias in the model. Finally, link test was performed to check if the model was well specified without over or under parameterization. 


\section{Results}

\section{Participants and participants demographic characteristics}

Out of the 380 participants sampled, 302 were successfully traced, accounting for $79.5 \%$ of the sample size (380). Among the 302 succesfully traced, 300 participants were enrolled into the study while two (02) refused to consent for the study. The mean age of the participants was 26.4 years ( $\mathrm{SD} \pm 6$, Range $=18-50)$, more than two-thirds $(68.3 \%)$ of the participants were below 30 years of age, $57.7 \%$ attained only primary education, $51.7 \%$ were never married and one-fifths (26.7\%) were divorced. Majority of the participants resided within Gulu (99.4\%), in urban setting (90.7\%), were Catholic (60.7\%), and originated from Gulu district (48.7\%) (Table 1).

\section{Table 1: Socio-demographic characteristics of FSWs in post-conflict Gulu District}

\begin{tabular}{|c|c|c|}
\hline Characteristic & Number & Percentage $(\%)$ \\
\hline \multicolumn{3}{|l|}{ Age (years) } \\
\hline$<30$ & 205 & 68.3 \\
\hline$\geq 30$ & 95 & 31.7 \\
\hline \multicolumn{3}{|l|}{ Education Level } \\
\hline None & 13 & 4.3 \\
\hline Primary education & 173 & 57.7 \\
\hline O-level education & 94 & 31.3 \\
\hline A-level and above & 20 & 6.7 \\
\hline \multicolumn{3}{|l|}{ Origin } \\
\hline Gulu District & 146 & 48.7 \\
\hline Other District within Northern Uganda & 125 & 41.6 \\
\hline Outside Northern Uganda & 29 & 9.7 \\
\hline \multicolumn{3}{|l|}{ Residence } \\
\hline Gulu District & 298 & 99.4 \\
\hline Other District within Northern Uganda & 1 & 0.3 \\
\hline Outside Northern Uganda & 1 & 0.3 \\
\hline \multicolumn{3}{|l|}{ Location of residence } \\
\hline Urban & 272 & 90.7 \\
\hline Rural & 28 & 9.3 \\
\hline \multicolumn{3}{|l|}{ Marital Status } \\
\hline Never married & 157 & 51.7 \\
\hline Cohabiting & 37 & 12.3 \\
\hline Married & 6 & 2.0 \\
\hline Divorced & 80 & 26.7 \\
\hline Widowed & 22 & 7.3 \\
\hline \multicolumn{3}{|l|}{ Religion } \\
\hline None & 18 & 6.0 \\
\hline Catholic & 182 & 60.7 \\
\hline Protestant & 48 & 16.0 \\
\hline Born Again & 34 & 11.3 \\
\hline Muslim & 16 & 5.3 \\
\hline Others & 2 & 0.7 \\
\hline
\end{tabular}




\section{Reasons for joining sex work}

Majority of the participants $(89.7 \%)$ mentioned poverty-related reasons as their main driving force for joining sex work of whom $51 \%$ joined sex work to earn money for survival, $22 \%$ were frustrated by financial hardship, $6.3 \%$ join sex work because of widowhood or divorce that had robbed them of financial support from their husbands, 5.7\%\% stated that their parents/guardians were not supportive, and $4.3 \%$ join sex work in order to look for school fees (Figure 1).

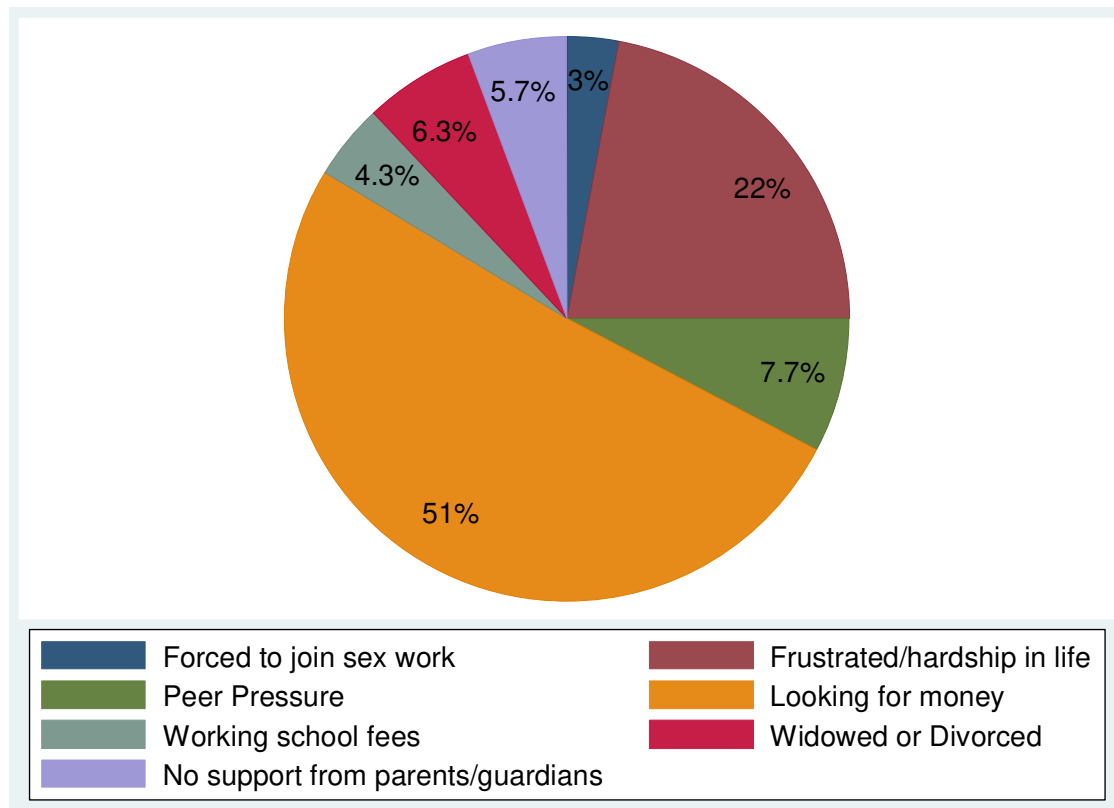

Figure 1: Showing reasons for joining sex work among FSWs operating in Gulu district Prevalence of MD among FSWs

In this study, the prevalence of MD among the FSWs stood at 47.7\%. Majority of the participants with MD (50.4\%) had severe depression (8-9 symptoms) and 40.6\% had moderate depression (67 symptoms). Meanwhile, the rest of the participants with MD (9.1\%) had mild depression (the bare minimum of 5 symptoms). The commonest symptoms of MD were: feeling consistently depressed (62.3\%), anhedonia (59.7\%) and being without energy (53\%). Meanwhile, the least common presenting symptoms of MD was feeling suicidal (16.4\%). However, when considering only participants with MD, one-third (34.3\%) of FSWs presented with suicidal ideation.

\section{Factors associated with MD among FSWs}

Results from bivariate analysis showed that several factors were significantly associated with MD. Factors like being psychologically stressed $(p<0.001)$, living with HIV/AIDS $(p<0.001)$, numbers of previous pregnancies $(p<0.001)$, age $(p<0.01)$, being verbally abused by client $(p<0.01)$, being physically abused by client $(p=0.02)$, and being raped by client $(p<0.05)$ all showed significant positive association with MD. Meanwhile, daily intake of alcohol $(p=0.01)$ and consistent use of condom $(p=0.04)$ both showed statistically significant negative association with MD.

In the multivariate logistic regression model, several factors including duration of sex work, income level, and condom use were controlled for. The results revealed that being psychologically 
stressed (aOR=11.0, 95\%CI: 5.77-20.86), living with HIV/AIDS (aOR=2.32, 95\%CI: 1.29-4.15), being verbally abused by the clients $(\mathrm{aOR}=2.27$, 95\%CI: $1.26-4.07)$, and being $30+$ years of age $(\mathrm{aOR}=1.95,95 \% \mathrm{CI}: 1.05-3.61)$ all had significant positive association with MD. Conversely, providing sexual services at clients' places (aOR=0.46, 95\%CI: $0.24-0.91)$, use of modern family planning method ( $\mathrm{aOR}=0.47,95 \% \mathrm{CI}$ : $0.25-0.87)$, and daily intake of alcohol (aOR=0.50, 95\%CI: 0.28-0.88) all showed significant negative association with MD ( $\underline{\text { Table 2) }}$.

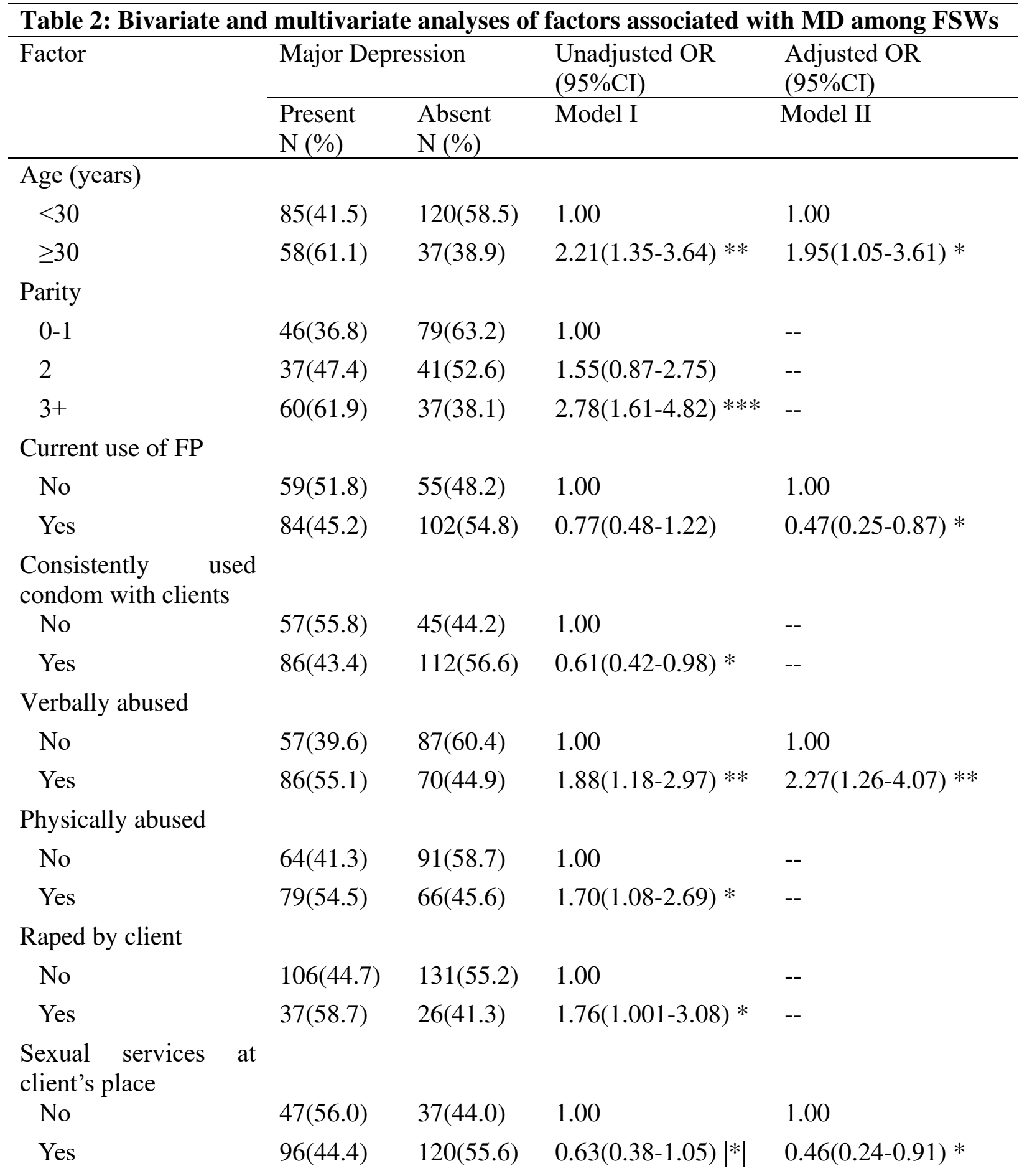


HIV Status

$\begin{array}{lllll}\text { HIV-Négative } & 65(37.6) & 108(62.4) & 1.00 & 1.00 \\ \text { HIV-Positive } & 78(61.9) & 48(38.1) & 2.70(1.68-4.33) * * * & 2.32(1.29-4.15) * *\end{array}$

Feeling stressed

No

18(16.1) 94(83.9) $\quad 1.00$

1.00

Yes

$125(66.5)$

63(33.5)

$10.4(5.75-18.66)$

$11.00(5.77-20.86)$

Alcohol intake

None or occasional

Always

$73(55.7)$

$58(44.3)$

1.00

1.00

$|*|=0.05<\mathrm{p}<0.2, *=\mathrm{p}<0.05, * *=\mathrm{p}<0.01, * * *=\mathrm{p}<0.001$

\section{Model Checking and Post Estimation Tests}

The model was assessed for outliers, influential points, sensitivity, specificity, specification error and goodness-of-of fit. The model performed very well across all above the regression diagnostic tests: graph of predicted residual showed that all the points lie between -3 and +3 meaning that there were no outliers and the points were evenly distributed across the central lines of residual equal to zero implying that the model fitted the data points well. Secondly, from the Linktest, the P-values associated with Hatsq $(\mathrm{p}=0.62)$ was not significant at $95 \% \mathrm{CI}$. This implies that there was no specification error and the model was well specified. Thirdly, the model fitted the data well since the observed and expected cell frequencies in the model were generally in good agreement with each other and the Hosmer-Lemeshow chi2 $(8)=3.74, p=0.88$ showed that the model had an excellent goodness-of-fit outcome since the P-value was very large. Lastly, the area under ROC curve was 0.83 and the sensitivity and specificity lines in the model intersect above 0.75 . This showed that the model had very high predictive power (figure 2).
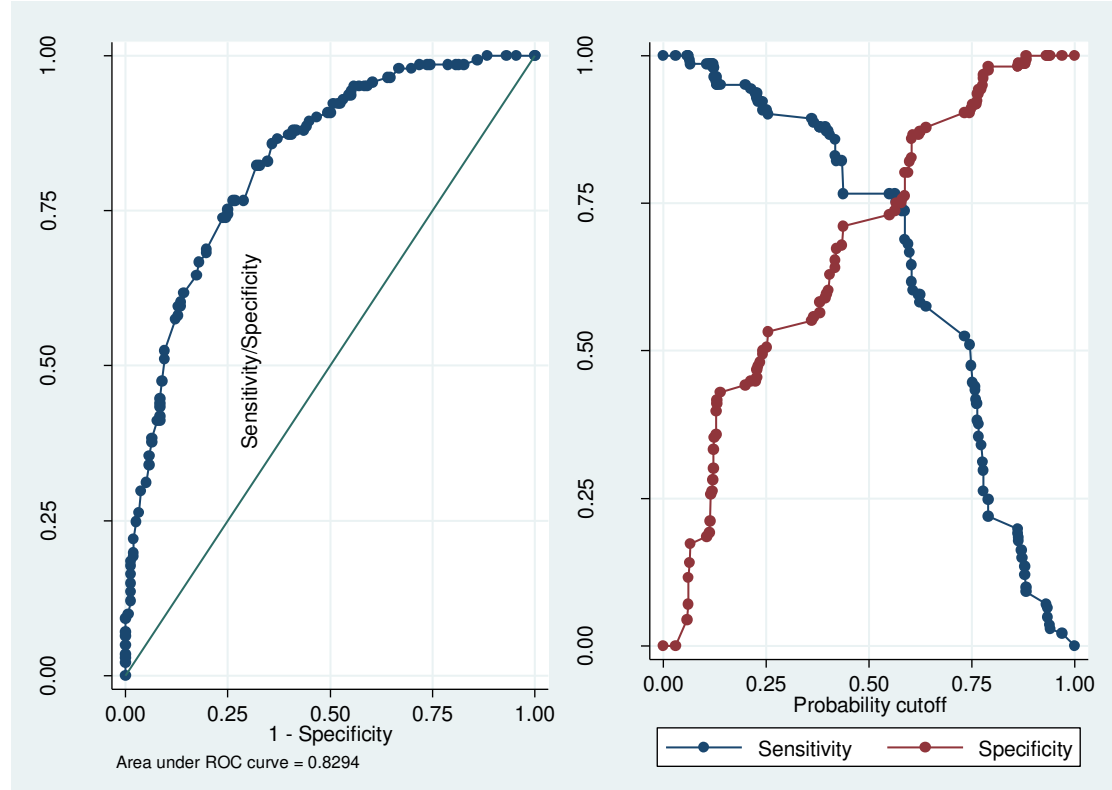

Figure 2: Showing area under ROC curve and predictive power of the regression model 


\section{Discussion}

The prevalence of MD among FSWs in post-conflict Gulu district was 47.7\%. This is well above the prevalence of MD in the general population of Gulu district (24.7\%), among women in the district (29.2\%) (27), among the FSWs in Southern India (29\%) (28) and China (31\%) (29), and the estimated global prevalence of depression (10.8\%) in conflict-affected settings (30). However, the current finding is comparable with the prevalence of depression among men who have sex with men (46.3\%) in the neighboring Tanzania (31) and conflict-affected South Sudan (50\%) (16). Majority of the cases of MD had either severe $(50.4 \%)$ or moderate depression $(40.5 \%)$. Studies that classify MD based on severity using numbers of depressive symptoms based on DSM-5 diagnostic criteria are extremely rare. One similar study, a national survey in the US, reported that similar proportion of Americans with MD had severe (49.5\%) [8-9 symptoms] and moderate depression (39.7\%) [6-7 symptoms] (26). The high proportion of FSWs suffering with moderateto-severe depression (89.2\%) implies that majority of FSWs with MD in the district require treatment for their depression as guided by previous recommendation (30). Moreover, urgent interventions are needed to address the high prevalence of MD among FSWs since up to one-third (34.3\%) of the FSWs with MD had suicidal ideation which pose serious risk to their lives.

At multivariate level, several factors were found to have significant positive association with MD. Verbal abuse of FSWs by their clients significantly increased MD in our study. Similar findings of increase risk of depression as a result of verbal abuse were reported among FSWs in Southern India (28) and among transgender population of Latina in Los Angeles (32). Likewise, living with HIV significantly increased MD among participants. Previous study noted that HIV significantly increase the risk of MD and that relationship between HIV and MD is mediated through more than 10 risk factors for depression (33). Additionally, being 30+ years of age (older age) was found to significantly increase MD. This finding is in agreement with previous researches that reported age dependent increase in the prevalence of MD in conflict-affected settings (6) (30). However, it is worth noting that in the general population, the prevalence of depression does not differ with age (34). This implies that any intervention to address MD prevention, treatment and rehabilitation should target the older FSWs especially those aged 30 years and above.

Furthermore, some three factors showed negative association with MD. These factors include providing sexual services at the client's place, use of modern family planning method and daily intake of alcohol. Providing sexual services at clients' places significantly decreased MD among FSWs. To the best of our knowledge no previous study ever reported on the relation between MD and FSW's provision of sexual services at clients' places. However, a study in Canada found that compared to Brothels, FSWs providing sexual services in the outdoor spaces/public spaces and informal indoor spaces were at an increased risk of developing mental health problems (35). Therefore, we postulated that the negative association between MD and sex work at clients' places could be because sex work at clients' places decrease client perpetrated GBV (36) which is a risk factor for MD. However, there is need for further studies to understand the mechanisms through which provision of sexual services at the clients' places protect FSWs from MD. Secondly, use of modern family planning method decreased the odds of MD among FSWs. One similar finding showed that the use of contraception reduces the occurrence of depression among women (37). While other studies reported that hormonal contraceptives did not have any significant effect on occurrence of major depression (38) (39). Yet another study reported mixed outcomes depending on type of contraception (40). For this current study, we postulate that effective family planning method protects FSWs from the risk of unwanted pregnancies and the anxiety associated with 
unwanted pregnancy especially when involved in condom-less sex with their clients. Lastly, daily intake of alcohol also reduced the odds of MDD in our study. It should be noted that there is a possibility of bi-directional relationship between MD and alcohol; either alcohol helped relieve depressive symptoms or because the factors surrounding alcohol intake like socializing make FSWs less likely to get MD. Our finding conflicts with previous finding showing alcohol as a risk factor for MD in the general population (27). However, two large longitudinal studies noted that daily intake of alcohol or alcohol use disorder did not have effect on the occurrence of MD (41) (42). Our finding may be explained by a study that indicated that drinking alcohol is a coping strategy for FSWs since they face several occupation risk of MD (43). It appears as if alcohol reduce the risk of MD, however, caution should be taken since alcohol use predisposes FSWs to condomless sex (44) to the extent of putting their sexual reproductive health at risk.

The very high prevalence of depression calls for the Ugandan government through the Ministry of Health and the district health officers and the development partners to put more resources to address the mental health needs of sex workers in post-conflict Gulu and the rest of conflictaffected settings in the countries. Secondly, in the light of the high prevalence of MD associated with risky sexual behaviour like condom-less sex and GBV, all stakeholders need to integrate mental health services for FSWs with other health care services that target HIV prevention among this most at risk population. This will help the country reduce new HIV infections and thus achieve the ambitious UNAIDS goals of ending HIV/AIDS by 2030. It was noted that HIV-positive FSWs were at increased odd of MD. Therefore, there should be a special program to provide HIV related counselling to this population in order to address HIV related factors that put HIV-positive FSWs at a higher risk of MD. Lastly, this study findings showed an unexpectedly negative association between daily alcohol intake and MD yet most previous evidences indicated that daily alcohol intake had positive association with MD. This contradiction needs clarification and calls for further studies using longitudinal studies to determine the causal relationship between MD and alcohol amongst this underserved population of key population especially in conflict-affected settings.

\section{Strengths and limitations of the study}

Our study had several strengths: unlike most previous studies among FSWs which use nonrandomized method of participants' selection, we utilized simple random sampling technique to select our sample. Thus, our sample was truly representative of the FSWs in post-conflict Gulu district. Secondly, the results from this study were derived from an optimal sample size of 300 FSWs that provided adequate power to detect statistical significance. Thirdly, unlike most previous studies which only screened for depressive symptoms, our study actually made diagnosis of MD based on DSM-5 using the MINI 7.00. Moreover, we were able to quantify the prevalence and severity of MD among FSWs in post-conflict Gulu district. Lastly, all the regression diagnostics tests showed that the multivariate logistic model performed very well in predicting factors associated with MD without failing any of the tests.

However, this study had limitations. The study was cross-sectional and only elicited association but not causations of MD with other factors. Secondly, the information collected may have be influenced by recall bias since FSWs were asked about their past. However, most of the information asked were for events within two weeks prior to data collection. Thus, the possibility for recall bias was reduced. Thirdly, some of the information implored, like those relating to sex work, were sensitive and difficult to respond to. However, the participants had friendly interview experience with the PI and the female research assistant who had developed close working 
relationships with the FSWs while providing HIV care, treatment and prevention services to the FSWs in region. Therefore, chances of information bias were greatly reduced. Lastly, some possible factors associated with MD like coping strategy and perceived social support were not directly explored. However, most of the other important confounders were included in the data collection tool and adjusted for.

\section{Conclusion}

The current study contributes new evidence toward understanding of the prevalence and factors associated with depression among FSWs in conflict-affected setting in a low-income context. This study underscores the high prevalence of major depression driven by multiple factors like the presence of psychosocial stressor, HIV infection, experiencing verbal abuse from clients and age. The high prevalence of major depression has serious and significant important public health ramifications at both the individual and societal levels. 


\section{List of abbreviation}

AIDS: Acquired Immunodeficiency Syndrome, ART: Antiretroviral Therapy, aOR: Adjusted Odd Ratio, CI: Confidence Interval, DSM-5: Diagnostic and Statistical Manual of Mental DisordersFifth Edition, FSW: Female Sex Worker, HIV: Human Immunodeficiency Virus, NGO: NonGovernmental Organization, MD: Major Depression, MINI: Mini-International Neuropsychiatric Interview, STI: Sexually Transmitted Infection, TASO: The AIDS Support Organization, UGX: Ugandan Shilling, uOR: Unadjusted Odd Ratio, UNAIDS: Joint United Nations Program on HIV and AIDS, WHO: World Health Organization

\section{Ethics approval and consent to participate}

Approval to carry out this study was sought from the Makerere University School of Public Health Higher Degrees, Research and Ethics Committee. Written informed consent were provided by each consenting participant. The privacy and confidentiality of each individual participant was maintained throughout all the processes of participant's enrollment into the study and data collection. During data presentation, only aggregated figures, not individual information, were presented in order to maintain confidentiality. Likewise, all the participants who met the diagnostic criteria for major depression as per DSM-5 guideline were linked to the Psychiatric Clinic of Gulu Regional Referral Hospital for further management.

\section{Consent for publication}

Not applicable

\section{Availability of data and materials}

The datasets used and/or analysed during the current study are available from the corresponding author on reasonable request.

\section{Competing interests}

The authors declare that they have no competing interests.

\section{Authors' contributions}

OS conceived and designed the study, collected and entered data, conducted data analysis and wrote the first draft of the manuscript. NMT, RN and AC conceived the study, supported data analysis and interpretation of findings and critically reviewed the draft manuscript. All authors approved the manuscript for publication. 


\section{References}

1. WHO. Depression fact-sheets. Geneva; 2018. Available from: https://www.who.int/newsroom/fact-sheets/detail/depression

2. Paykel E. Depression: Major problem for public health. Epidemiol Psichiatr Soc. 2006;15(1):4-10.

3. Vigo D, Thornicroft G, Atun R. Estimating the true global burden of mental illness. Lancet Psychiatry. 2016;3(Februrary):171-8.

4. Luppa M, Heinrich S, Angermeyer C, König H, Riedel-Heller S. Cost-of-illness studies of depression: A systematic review. J Affect Disord. 2007;98(1):29-43.

5. Prince M, Patel V, Saxena S, Maj M, Maselko J, Phillips MR, et al. No health without mental health. Lancet. 2007;370(9590):859-77.

6. Senarath U, Wickramage K, Peiris S. Prevalence of depression and its associated factors among patients attending primary care settings in the post-conflict Northern Province in Sri Lanka: A cross-sectional study. BMC Psychiatry. 2014;14(1):1-10.

7. Ovuga E, Boardman J, Wasserman D. The prevalence of depression in two districts of Uganda. Soc Psychiatry Psychiatr Epidemiol. 2005;40(6):439-45.

8. Rössler W, Koch U, Lauber C, Hass A-K, Altwegg M, Ajdacic-Gross V, et al. The mental health of female sex workers. Acta Psychiatr Scand. 2010 Aug 1;122(2):143-52.

9. Odabaşı AB, Şahinoglu S, Genç Y, Bilge Y. The Experiences of Violence and Occupational Health Risks of Sex Workers Working in Brothels in Ankara. 2012;(July 2010):26-8.

10. WHO. Depression and Other Common Mental Disorders Global Health Estimates. Vol. 48. Geneva: World Health Organization; 2017. 1-20 p.

11. Sánchez-Fuentes M, Santos-Iglesias P, Sierra J. A systematic review of sexual satisfaction. Int J Clin Heal Psychol. 2014;14(1):67-75.

12. Baldwin D, Foong T. Antidepressant drugs and sexual dysfunction. Br J Psychiatry. 2013;202(6):396-7.

13. Sarkar N. Barriers to condom use. Eur J Contracept Reprod Heal Care. 2008 Jan $1 ; 13(2): 114-22$.

14. Hull MW, Montaner JSG. HIV treatment as prevention: The key to an AIDS-free generation. J Food Drug Anal. 2013;21(4 SUPPL.):S95-101.

15. Dévieux JG, Jean-Gilles M, Rosenberg R, Beck-Sagué C, Attonito JM, Saxena A, et al. Depression, Abuse, Relationship Power and Condom Use by Pregnant and Postpartum Women with Substance Abuse History. AIDS Behav. 2016;20(2):292-303.

16. Roberts B, Damundu E, Lomoro O, Sondorp E. Post-conflict mental health needs: A crosssectional survey of trauma, depression and associated factors in Juba, Southern Sudan. BMC Psychiatry. 2009;9:1-10.

17. Farhood L, Dimassi H, Strauss N. Understanding Post-Conflict Mental Health: Assessment of PTSD, Depression, General Health and Life Events in Civilian Population One Year after the 2006 War in South Lebanon. J Trauma Stress Disord Treat. 2013;02(02):1-8.

18. Ateenyi F, Linnete D. Legal regulation of sex work in uganda : exploring the current trends and their impact on the human rights of sex workers. Kampala; 2016.

19. Sheehan V. Depression: underdiagnosed, undertreated, underappreciated. Manag Care. 2004 Jun;13(6 Suppl Depression):6-8.

20. Gulu District. The Republic of Uganda: Gulu district Local government Report. Gulu; 2013.

21. Apodaca K, Doshi RH, Ogwal M, Kiyingi H, Aluzimbi G, Musinguzi G, et al. CaptureRecapture Among Men Who Have Sex With Men and Among Female Sex Workers in 11 Towns in Uganda. JMIR Public Heal Surveill. 2019;5(2):e12316. 
22. Isreal GD. Determining Sample Size. Vol. PEOD6, Determining Sample Size. 1992. p. 13. Available from: http;//edis.ifas.ufl.edu.

23. Ortblad K, MD K, Ngabirano T, Nakitende A, Harling G, Haberer J, et al. The Effect of HIV Self-Testing Delivery Models on Female Sex Workers' Sexual Behaviors: A Randomized Controlled Trial in Urban Uganda. AIDS Behav. 2019;23(5):1225-39.

24. Sheehan D., Janava J., Sheehan KH., Baker R., Knapp E SM. Mini international neuropsychiatric interview-version 7.0.0. 2014.

25. American Psychiatric Association. American Psychiatric Association Diagnostic and Statistical Manual of Mental Disorders. 5th ed. Arlington, VA; 2013.

26. Hasin DS, Sarvet AL, Meyers JL, Saha TD, Ruan WJ, Stohl M, et al. Epidemiology of Adult DSM-5 Major Depressive Disorder and Its Specifiers in the United States. JAMA psychiatry . 2018 Apr 1;75(4):336-46.

27. Mugisha J, Muyinda H, Malamba S, Kinyanda E. Major depressive disorder seven years after the conflict in northern Uganda: Burden, risk factors and impact on outcomes (The Wayo-Nero Study). BMC Psychiatry. 2015;15(1):1-12.

28. Patel SK, Ganju D, Prabhakar P, Adhikary R. Relationship between mobility, violence and major depression among female sex workers: a cross-sectional study in southern India. BMJ Open. 2016;6(9):e011439.

29. Yang Q, Operario D, Zaller N, Huang W, Dong Y, Zhang H. Depression and its correlations with health-risk behaviors and social capital among female migrants working in entertainment venues in China. PLoS One. 2018;13(2):1-13.

30. Charlson F, van Ommeren M, Flaxman A, Cornett J, Whiteford H, Saxena S. New WHO prevalence estimates of mental disorders in conflict settings: a systematic review and metaanalysis. Lancet . 2019;394(10194):240-8.

31. Ahaneku H, Ross M, Nyoni J, Selwyn B, Troisi C, Mbwambo J, et al. Depression and HIV risk among men who have sex with men in Tanzania. AIDS Care. 2016 Mar 24;28(sup1):140-7.

32. Bazargan M, Galvan F. Perceived discrimination and depression among low-income Latina male-to-female transgender women. BMC Public Health. 2012;12(1):1.

33. Ayano G, Solomon M, Abraha M. A systematic review and meta-analysis of epidemiology of depression in people living with HIV in east Africa. BMC Psychiatry. 2018;18(1):1-13.

34. Brody DJ, Pratt LA, Hughes JP. Prevalence of Depression Among Adults Aged 20 and Over: Uited States, 2013-2016. 2018.

35. Puri N, Shannon K, Nguyen P, Goldenberg SM. Burden and correlates of mental health diagnoses among sex workers in an urban setting. 2017;1-9.

36. Schwitters A, Swaminathan M, Serwadda D, Muyonga M, Shiraishi RW, Benech I, et al. Prevalence of Rape and Client-Initiated Gender-Based Violence Among Female Sex Workers: Kampala, Uganda, 2012. AIDS Behav. 2015;19(1):68-76.

37. Keyes KM, Cheslack-Postava K, Westhoff C, Heim CM, Haloossim M, Walsh K, et al. Association of hormonal contraceptive usewith reduced levels of depressive symptoms: A national study of sexually activewomen in the united states. Am $\mathrm{J}$ Epidemiol. 2013;178(9):1378-88.

38. Kessler RC. Epidemiology of women and depression. J Affect Disord. 2003;74(1):5-13.

39. O'Connell K, Davis AR, Kerns J. Oral contraceptives: side effects and depression in adolescent girls. Contraception. 2007;75(4):299-304.

40. Roberts TA, Hansen S. Association of Hormonal Contraception with depression in the postpartum period. Contraception. 2017;96(6):446-52.

41. Wang J, Patten SB. Alcohol consumption and major depression: Findings from a follow-up 
study. Can J Psychiatry. 2001;46(7):632-8.

42. Haynes JC, Farrell M, Singleton N, Meltzer H, Araya R, Lewis G, et al. Alcohol consumption as a risk factor for non-recovery from common mental disorder: Results from the longitudinal follow-up of the National Psychiatric Morbidity Survey. Psychol Med. 2008;38(3):451-5.

43. Ulibarri MD, Hiller SP, Lozada R, Rangel MG, Stockman JK, Silverman JG, et al. Prevalence and characteristics of abuse experiences and depression symptoms among injection drug-using female sex workers in mexico. J Environ Public Health. 2013;2013.

44. Hong Y, Li X, Fang X, Zhao R, Yang Hong, Xiaoming Li, Xiaoyi Fang and RZ. Depressive symptoms and condom use with clients among female sex workers in China. Sex Heal. 2007;4(2):99-104. 
Figures

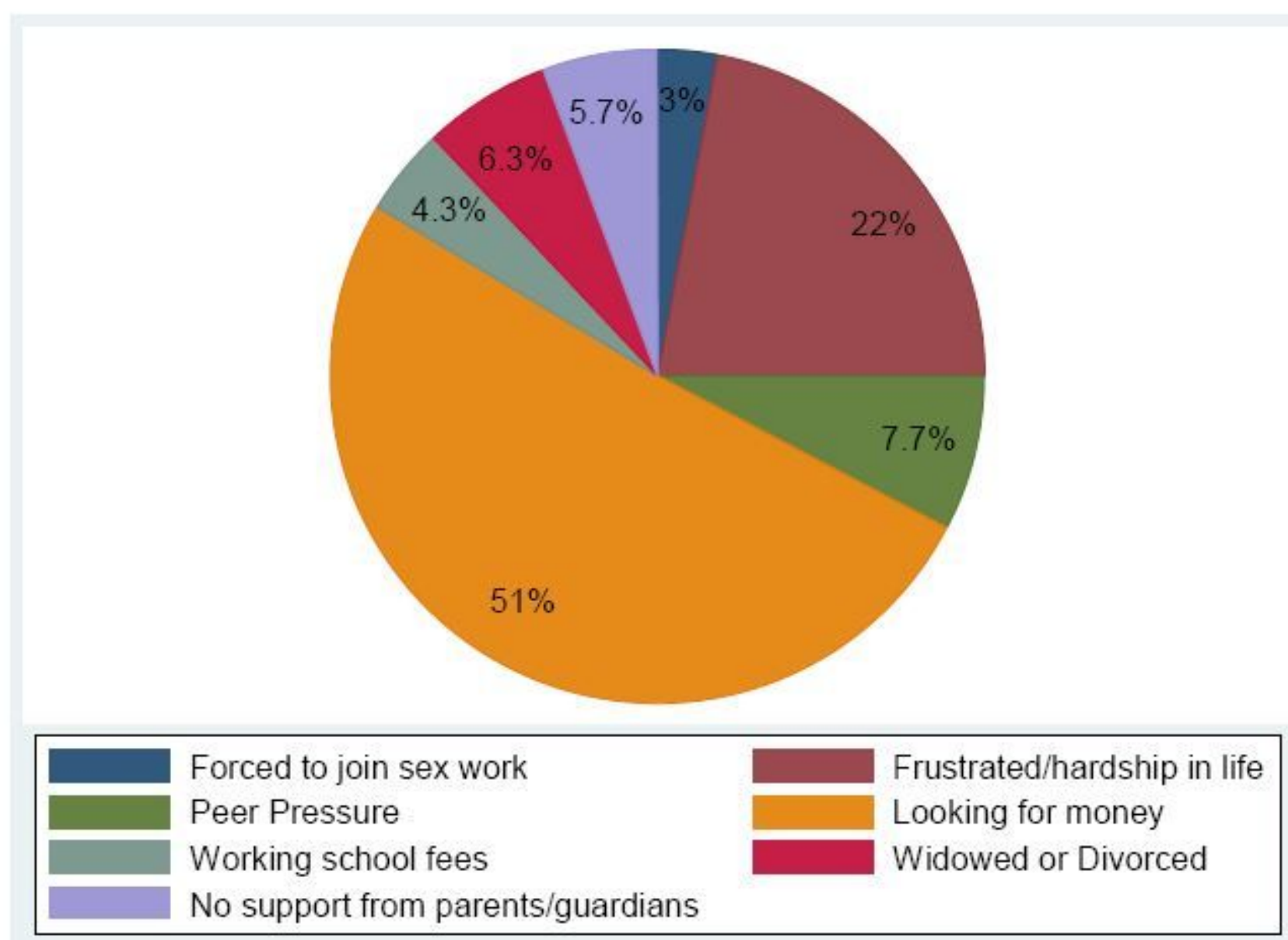

\section{Figure 1}

Showing reasons for joining sex work among FSWs operating in Gulu district 

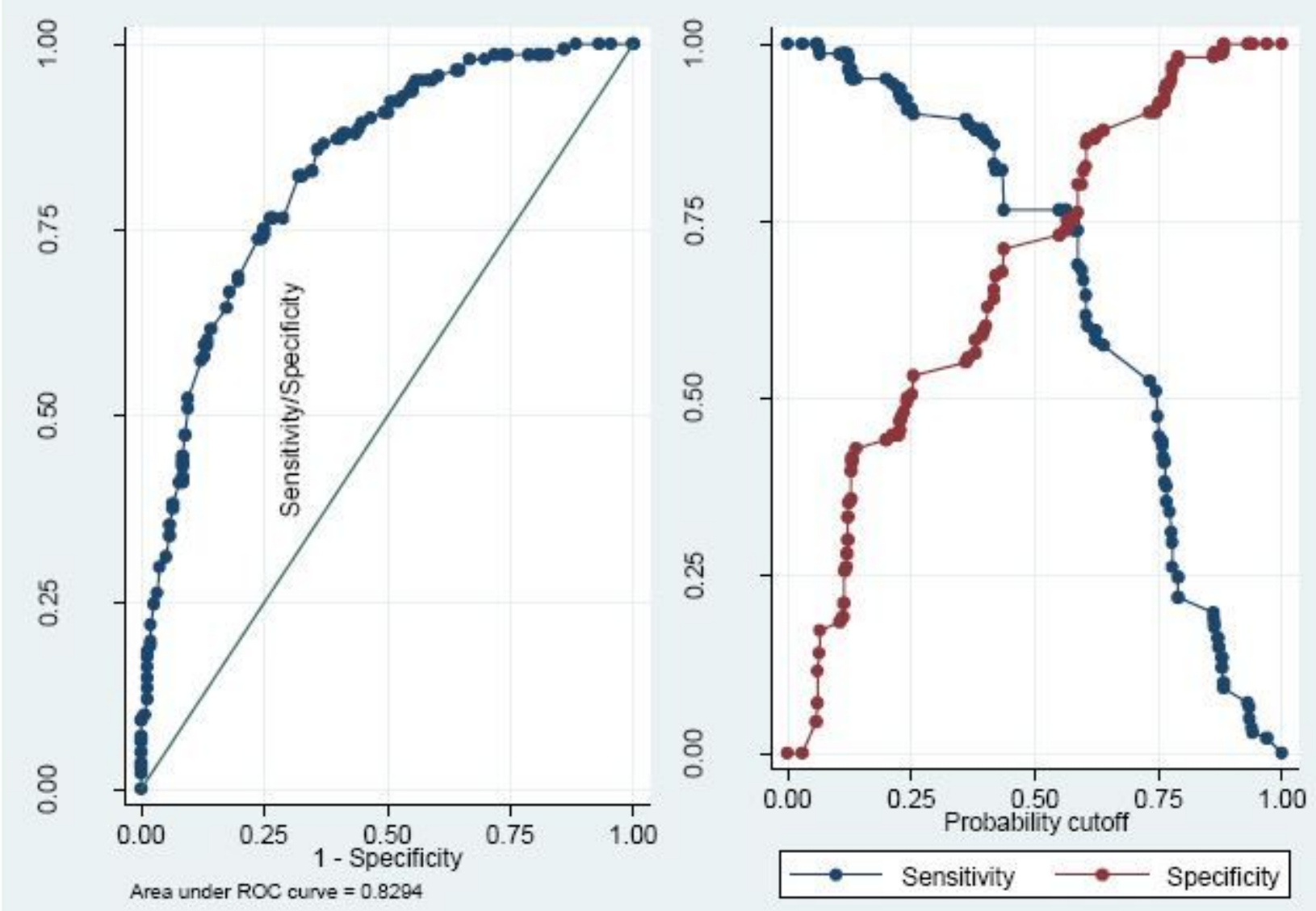

Figure 2

Showing area under ROC curve and predictive power of the regression model 